\title{
Heat Exchanger Fouling and Estimation of Remaining Useful Life
}

\author{
Tutpol Ardsomang ${ }^{1}$, J. Wesley Hines ${ }^{2}$, and Belle R. Upadhyaya ${ }^{2}$ \\ ${ }^{1}$ Department of Industrial and Systems Engineering, University of Tennessee, Knoxville, TN 37996, USA \\ tardsoma@utk.edu \\ ${ }^{2}$ Department of Nuclear Engineering, University of Tennessee, Knoxville, TN 37996-2300, USA \\ jhines@utk.edu \\ bupadhya@utk.edu
}

\begin{abstract}
One of the challenges in data-driven prognostics is the availability of degradation data for application to prognostic methods. In real process management settings, failure data are not often available due to the high costs of unplanned breakdowns. This research presents a data-driven (empirical) modeling approach for characterizing the degradation of a heat exchanger (HX) and to estimate the Remaining Useful Life (RUL) of its design operation. The Autoassociative Kernel Regression (AAKR) modeling was applied to predict the effect of fouling on the heat transfer resistance. The result indicates that AAKR model is an effective method to capture the HX fouling in the dynamic process. The AAKR residuals were fused to develop a prognostic parameter which was used to develop a General Path Model (GPM) with Bayesian updating. The results demonstrate the successful application of this approach for the heat exchanger RUL prediction.
\end{abstract}

\section{INTRODUCTION}

Fouling is defined as the formation of unwanted material deposition on the heat transfer surface. It occurs naturally in heat transfer processes across several types of industries. The fouling problem exists in more than 90 percent (Steinhagen R, 1993) of heat exchangers. The consequences of fouling accumulation include not only the economic loss, but heat transfer efficiency degradation, high flow resistance and pressure drops, and increased safety hazards, such as overheating of the HX surface.

The fouling problem has been the topic of intensive research by several groups. Several methods on the fouling prediction of heat exchanger (HX) have been continuously

Tutpol Ardsomang et al. This is an open-access article distributed under the terms of the Creative Commons Attribution 3.0 United States License, which permits unrestricted use, distribution, and reproduction in any medium, provided the original author and source are credited. proposed. Since the fouling progression is a complicated process, influenced by many parameters and not well understood, the available mathematical models do not cover all forms of fouling mechanisms. The lack of physical understanding of the fouling process dynamics is still a crucial issue that needs to be further investigated.

In recent years, empirical modeling approaches such as the Artificial Neural Network (ANN), Support Vector Machine (SVM) and other statistical techniques have been widely applied in several types of industrial processes. With the ability to learn the different operating conditions of the data and to develop the model without any physical understanding of the process, the empirical modeling approaches have become more interesting and promising for the complicated fouling problem. Several publications, that address the issue of heat exchanger fouling prediction, are available (Vasilios, 2012; Lingfang, 2008; Upadhyaya et al., 2004; Ingimundardóttir, 2009). In this project, an AAKR model is selected to develop the fouling prediction model. AAKR is a non-linear, non-parametric model that uses kernel regression to interpolate historical data stored in a memory matrix. The prediction results shows that an AAKR model can be applied appropriately to both dynamic and static heat exchanger processes.

The fouling prediction using various types of empirical modeling has been successfully studied. However, calculating the Remaining Useful Life (RUL) of the HX when the fouling is first noticeable has not yet been fully explored. In this project, the General Path Model (GPM) (Hines and Coble, 2010) is introduced for the heat exchanger's RUL prediction.

The GPM is developed using historical degradation measurements. The degradation path is expressed as the measure of degradation from the time when a fault first occurs to the end of life, which is usually indicated by exceeding a predetermined critical threshold. This threshold 
can be defined by a performance specification or engineering judgment. It is assumed that component degradation path can be described by some underlying parametric model and there is a unique degradation path for each individual component. The model parameters can be updated using new individual observations and applying Bayesian updating techniques, which allows both current observation and past knowledge to be considered in the model fitting.

The RUL prediction of early-detected fouling in a heat exchanger is performed using the GPM with Bayesian updating techniques. The historical failed degradation paths which were simulated based on the asymptotic fouling behavior were used to develop the model. The unfailed degradation data were also simulated to test the performance and accuracy of the GPM predictions. The results show that the GPM with Bayesian updating gives an effective model to predict the RUL of the heat exchanger.

All analysis in this study was performed using the MATLAB $^{\mathrm{TM}}$ software. The AAKR models were developed, tested and validated using the Process and Equipment Monitoring (PEM) toolbox (Hines and Garvey, 2006), developed by the University of Tennessee. The RUL predictions of heat exchanger were developed, using the MATLAB-based Process and Equipment Prognostic (PEP) toolbox (Hines and Garvey, 2011).

\section{BACKGROND}

This section introduces the basic mechanisms of heat excahnger fouling, HX physical model, AAKR and GPM with Bayesian updating.

\subsection{Heat Exchanger Fouling}

Fouling is the terminology generally used to describe the accumulation and formation of unwanted material on the material phase interface, which is the cause of the heat transfer capacity deterioration. Fouling is one of the most important problems of the heat transfer equipment such as heat exchangers, boilers, and steam generators. There is considerable interest in this subject because of the detrimental impact on the economy in various industries.

The fouling process is a result of two processes: a deposition of contamination onto the tube walls and a removal of deposition from the tube walls. The rate of fouling deposition growth (fouling resistance or fouling factor, $R_{f}$ ) can be calculated as the difference between the deposition and removal rates as shown in Equation (1).

$$
R_{f}=\Phi_{d}-\Phi_{r}
$$

The fouling behavior is classified into four categories (Epstein, 1988), as indicated in Figure 1. The delay time $t_{d}$ is the initial time period where no fouling occurs. The $t_{d}$ is unpredictable since it appears to be random in nature.
However, $t_{d}$ is generally shorter after the first fouled surface is cleaned. The plots in Figure 1 represent four different types of fouling mechanisms.

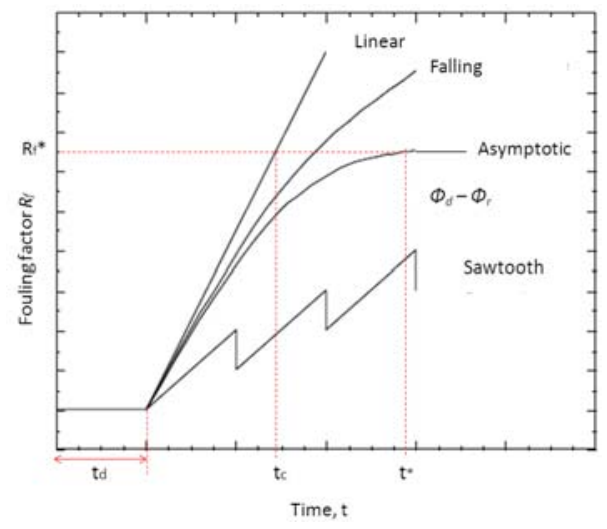

Figure 1. Fouling Curve (Epstein, 1988)

- Linear fouling indicates the constant deposition rates which the difference between $\Phi \mathrm{d}$ and is $\Phi \mathrm{r}$ is constant.

- Falling fouling curve indicates the mass of deposit increase with time but not linearly and does not reach the steady state.

- Asymptotic fouling curve is the most common in the industrial process. The pure particulate fouling also falls into this mode. It indicates the rate of fouling gradually decreasing over time and reaching a steady state eventually, when $\Phi_{d}$ is equal to $\Phi r$. The fluid velocity causes a shear stress at the fouling layer that removes some of the particulates. As the fouling layer becomes thick, the fluid velocity increases, thus increasing the rate of removal of the deposit. The thickness of the final steady-state fouling layer is inversely proportional to the original velocity (Nesta, 2004).

- Sawtooth indicates generally increasing trend punctuated by short periods of decreasing trend due to periodic shedding of fouling deposits

For the asymptotic fouling behavior, the fouling resistance, $R_{f}$, can be expressed by

$$
R_{f}=R_{f}^{*}\left(1-e^{-\beta_{c} \theta}\right)
$$

where $R_{f} *$ is the asymptotic fouling resistance which $\Phi_{d}$ is equal to $\Phi r, \beta c$ is a decay rate and $\theta$ is the time or usage parameter.

\subsection{Heat Exchanger Physical Model}

The shell and tube design is one of the simplest and popular HX types, which can be found in most process plants. It consists of a series of tubes, called the tube bundle, which contain the fluid that must be either heated or cooled. The second fluid flows over the tube in the shell side which can either supply or remove the heat. Figure 2 is a diagram of a 
simple shell and tube heat exchanger which was used in this study.

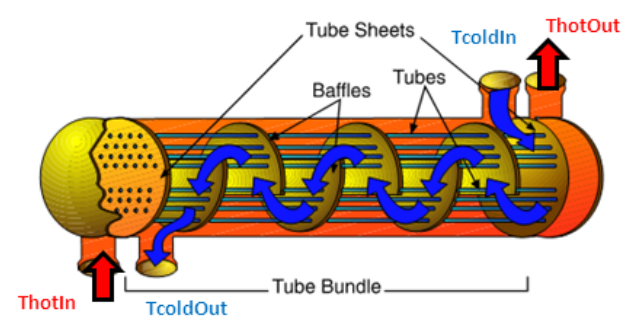

Figure 2. Shell and Tube Heat Exchanger

The traditional heat balance model is utilized based on the assumption that the amount of heat given up by the hot fluid is equal to the amount of heat received by the cold fluid, as shown in the Equation (3).

$$
\dot{Q}=\dot{m}_{h} C_{h} \Delta T_{h}=\dot{m}_{c} C_{c} \Delta T_{c}
$$

where $\dot{m}$ is mass flow rate, $C_{p}$ is specific heat capacity and $\Delta \mathrm{T}$ is temperature difference. In general, there are two physical models which represent the heat exchanger: the Log Mean Temperature Difference (LMTD) method and the Effectiveness-NTU method.

The heat transfer can also be expressed by

$$
\dot{Q}=U A \Delta T_{L M T D}
$$

Where $U$ is the overall heat transfer coefficient, $A$ is the heat transfer area, $\Delta T_{L M T D}$ is the log-mean temperature difference for the heat exchanger, and is given by (Holman, 1981)

$$
L M T D=\Delta T_{L M T D}=\frac{\Delta T_{1}-\Delta T_{2}}{\ln \left(\frac{\Delta T_{1}}{\Delta T_{2}}\right)}
$$

If the heat exchanger is the concurrent flow type:

$$
\Delta T_{1}=T_{h_{\text {in }}}-T_{c_{\text {in }}}, \Delta T_{2}=T_{h_{\text {out }}}-T_{c_{\text {out }}}
$$

For the counter current flow heat exchanger:

$$
\Delta T_{1}=T_{h_{\text {in }}}-T_{c_{\text {out }}}, \Delta T_{2}=T_{h_{\text {out }}}-T_{c_{\text {in }}}
$$

The reciprocal of UA is called the overall thermal resistance, which increases in proportion to the fouling deposition amount. From Equation (4), the overall thermal resistance can be derived as

$$
\frac{1}{U A}=\frac{\Delta T_{L M T D}}{\dot{m}_{h} C_{h} \Delta T_{h}}=\frac{\Delta T_{L M T D}}{\dot{m}_{c} C_{C} \Delta T_{C}}
$$

The effectiveness-NTU (Holman 1981) method is used when the information is insufficient to calculate the logmean temperature (LMTD); for instance, when the fluid output temperatures are unknown. Based on the effectiveness of the heat exchanger, it can be defined as the rate between the actual heat transfer and the maximum possible heat transfer which can be hypothetically obtained in a counter-flow heat exchanger.

$$
e=\frac{Q}{Q_{\max }}
$$

It is noted that the fluid will experience the maximum possible temperature difference, which is the difference between inlet of primary side and the inlet of secondary side $\left(T_{h_{\text {in }}}-T_{c_{\text {in }}}\right)$.

$$
\begin{aligned}
\dot{Q}_{\text {max }} & =\left(\dot{m} \dot{\times} c_{p}\right)_{\text {min }}\left(T_{h_{i n}}-T_{c_{i n}}\right) \\
& =\dot{m} C_{\min }\left(T_{h_{i n}}-T_{c_{i n}}\right)
\end{aligned}
$$

$C_{\min }$ is the heat capacity rate computed by the multiplication of mass flow rate and minimum specific heat, which is either of the hot or the cold fluid. From equations (5), (8) and (9), the effectiveness equation is

$e=\frac{C_{h}\left(T_{h_{\text {in }}}-T_{h_{\text {out }}}\right)}{C_{\min }\left(T_{h_{\text {in }}}-T_{c_{\text {out }}}\right)} \quad$ or $e=\frac{C_{c}\left(T_{c_{\text {in }}}-T_{c_{\text {out }}}\right)}{C_{\min }\left(T_{h_{\text {out }}}-T_{c_{\text {in }}}\right)}$

Where $C_{h}$ and $C_{c}$ are the heat capacity rate of hot and cold side, respectively, and are computed from the multiplication of mass flow rate and specific heat capacity. The Number of Thermal Units (NTU) is expressed as

$$
N T U=\frac{U A}{C_{\min }}
$$

\subsection{Autoassociative Kernel Regression}

AAKR is a type of kernel regression modeling which is a non-parametric, empirical modeling technique that uses historical, fault-free observations to correct any errors present in current observations. The term "Autoassociative" means that the model inputs and outputs are the same variables. Autoassociative is the common empirical architecture, which all variables input to the model are estimated. It is useful for monitoring equipment or system with a high degree of correlation between variables.

Basically, an AAKR is constructed by putting the non-faulty data into the memory matrix. An AAKR model will learn the relationships from all the variables. When the new data come in, the model will interpolate between those new data 
and the memory matrix using kernel regression. If the inputs contain the error either by noise, instrument drift or process drift, the model is expected to predict what the normal value should be. The residual between the predicted and measured values can be monitored to detect anomalies in instrument channels and the process.

In AAKR a set of new measurements and a set of prototypical measurements are compared based on distance operator. First, the exemplar or memory vectors used to develop the empirical model are stored in a matrix $X$, where $X_{i j}$ is the $i^{\text {th }}$ observation of the $j^{\text {th }}$ variable. For $n_{m}$ observations of $p$ process variables, this matrix is expressed as

$$
\mathbf{X}=\left[\begin{array}{cccc}
X_{1,1} & X_{1,2} & \cdots & X_{1, p} \\
X_{2,1} & X_{2,2} & \cdots & X_{2, p} \\
\vdots & \vdots & \ddots & \vdots \\
X_{n_{m}, 1} & X_{n_{m}, 2} & \cdots & X_{n_{m}, p}
\end{array}\right]
$$

A query vector is the vector of process variable measurements represented by a $1 x p$ vector

$$
\mathrm{x}=\left[\begin{array}{llll}
\mathrm{x}_{1} & \mathrm{x}_{2} & \ldots & \mathrm{x}_{\mathrm{p}}
\end{array}\right]
$$

The distance between a query vector and each of the memory vectors is computed. The most common function is the Euclidian distance

$$
d_{j}=\sqrt{\sum_{i=1}^{n}\left(X_{j, i}-x_{i}\right)^{2}}
$$

This calculation is repeated for each of the $n_{m}$ memory vectors, resulting in an $n_{m} \times 1$ matrix of distances $(d)$. The distances are transformed to similarity measures used to determine weights by evaluating the Gaussian kernel with a bandwidth, $h$. The bandwidth is optimized using a cross validation technique to minimize the prediction error. This regularization is necessary for ill-posed problems to minimize prediction noise and improve repeatability [Hines 2005].

$$
w_{i}=K_{j}(d, h)=\frac{1}{\sqrt{2 \pi h^{2}}} \exp \left(\frac{-d_{j}^{2}}{2 h^{2}}\right)
$$

After achieving the optimal $h$ bandwidth, the prediction $\hat{y}_{p}$ is obtained by a weighted linear $\left(W_{i}\right)$ combination of the similar memory vectors expressed by the equation

$$
y_{p}=\frac{\sum_{i=1}^{n_{m}} w_{i} \cdot x_{i}}{\sum_{i=1}^{n_{m}} w_{i}}
$$

\subsection{General Path Model with Bayesian Updating}

Since the degradation of the heat exchanger performance is measurable, the condition based prognostics is the most appropriate approach. The GPM is an example of degradation modeling developed by Lu and Meeker in 1993. GPM is based on the available degradation measurements to estimate Time to Failure (TTF) distribution. In fact, useful information underlying in degradation measurements may result in a better reliability prediction. The GPM analysis begins with the assumption that individual equipment will fall into the same underlying functional form of the degradation path under the specific failure mode.

Basically, degradation measurements show the degradation paths (or degradation signals) to the end of life which is usually indicated by the crossing of a predetermined critical threshold. However, it is not necessary that all units have to be run to failure, unfailed or right censored data also contain useful information for GPM prediction. Another assumption for GPM is that component degradation can be described by some underlying parametric model, based upon physical models or from historical degradation data, and there is a unique degradation path for each individual component. To estimate failure times using a GPM, degradation paths need to be extrapolated to the failure threshold. Then the observed degradation paths will form the TTF distribution. The degradation of the $i^{\text {th }}$ unit at time $t_{j}$ is expressed by equation 18 .

$$
y_{i j}=\eta\left(t_{j}, \Phi, \Theta_{i}\right)+\varepsilon_{i j} \quad i=1,2,3, \ldots . n
$$

where $\eta$ is a GPM function, $t_{j}$ is time of the $j^{\text {th }}$ measurement or inspection, $\Phi$ is a vector of fixed-effect parameters, $\theta_{i}$ is a vector of random effects parameters for individual $i^{\text {th }}$ component, and $\varepsilon_{i j}$ is assumed the normal distribution with mean zero and the standard measurement error term $\mathrm{N}\left(0, \sigma=\varepsilon^{\wedge}\right)$. The model parameters are estimated from the historical data. This degradation path model, $y_{i}$, can be extrapolated to the failure threshold to estimate the component's time of failure.

The Bayesian technique can be combined with a GPM model to predict the RUL. Bayesian updating is a method that allows the prior information to combines with the new observations to update model parameter predictions. This method will allow both current observation and past knowledge to be considered in model fitting

A linear regression model, $Y=\beta X$, is considered to be the simplest approach to generate the model parameters. $\beta$ is the vector of parameters:

$$
\beta=\left(X^{T} \sum_{y}^{-1} X\right)^{-1} X^{T} \sum_{y}^{-1} Y
$$

Where $X$ is matrix of time, $Y$ is vector of degradation measure and $\sum_{y}$ is the variance-covariance noise matrix, which represents the accuracy of each entry in the Y-vector. 
In fact, the linear regression model is linear-in-parameters which can be populated with any function of degradation measures such as quadratic, exponential, sinusoidal, etc.

To include Bayesian updating, the prior information (model parameter) is treated as one additional data point to the Ordinary Least Square (OLS) solution, matrix $X$ is appended with an identity matrix $I_{k}$ and matrix $Y$ is appended with a priori value of the model parameter. The variancecovariance matrix is also added with a final row and column of zeros, with the variance of a priori information in the diagonal element, see the equation 20

$$
\mathrm{y}_{*}=\left[\begin{array}{c}
\mathrm{y} \\
\mathrm{b}_{0}
\end{array}\right], X_{*}=\left[\begin{array}{l}
X \\
I_{k}
\end{array}\right], \Sigma_{*}=\left[\begin{array}{cc}
\Sigma_{\mathrm{y}} & 0 \\
0 & \Sigma_{\mathrm{b}}
\end{array}\right]
$$

It is assumed that the noise in the degradation measurements is constant and uncorrelated across observations of $y$. This allows the variance-covariance matrix to be a diagonal matrix consisting of noise variance estimates and a priori knowledge variance estimates, which simplified computer implementation. After a priori knowledge is used in conjunction with $\mathrm{n}$ current data observation, the posterior estimated parameters become the new estimated parameters if more new data were obtained. The variance of the new data is estimated as

$$
\sigma_{\text {post }}^{2}=\left(\frac{1}{\sigma_{\text {prior }}^{2}}+\frac{n}{\sigma_{y}^{2}}\right)^{-1}
$$

There are two pieces of information, the prior and the data, used to form the posterior estimation. The weighting of these information depend on the variance of the prior such as the variance or uncertainty of the data and the amount of data. In other words, the prior $b_{0}$ will have large weighting if the variance of the prior is small. However, the data will be weighted more heavily when new data are collected.

\section{EXPERIMENTAL SETUP}

The current experimental setup was designed based on the previous experiment (Upadhyaya et al., 2004). The heat exchanger test bed is a simple two sided loop system. The exchanger, API Basco HT, is a shell-and-tube type exchanger with brass shell, internal brass tube sheets, and 64 $\mathrm{x} 1 / 4$ "copper tubes. The hot fluid passes through the tube while cold fluid passes through the shell. A 15-gallon tank holds the contaminated water used to accelerate the fouling process. A drain system utilizing a ball valve is placed between the tank and pump inlet to facilitate draining the system for cleaning. Three 1500 watt immersion heaters are inserted in the tank to generate heat up to 4,000 Watt into the water. A $0.5 \mathrm{HP}$ centrifugal pump is used to pump water through the closed loop with the maximum flow rate of 40 GPM at head pressure $20 \mathrm{ft}$ and 5 GPM at $80 \mathrm{ft}$ as the minimum. Figure 3 is a photograph of the heat exchanger test bed.

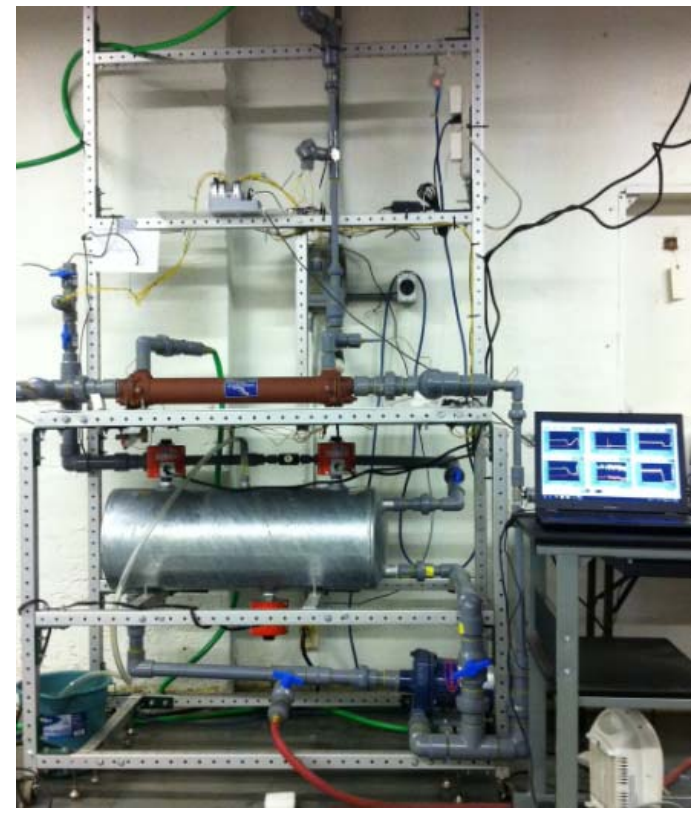

Figure 3. Heat Exchanger Test Bed.

Referring to the Piping and Instrument diagram (P\&ID) in Figure 4, the hot water is first pumped to a T-connector to split the water flow. One is the bypass pathway. The main function is to control pressure by $\mathrm{XV}-2$. The second pathway is the main line to the heat exchanger and return to the tank. The flow control valve FV-1, needle type, is installed to control the flow in the main line where the flow rate is measured by the turbine flow meter with $\mathrm{F}$ to $\mathrm{V}$ converter (Fhot). The water flow rate ranges between 0 to 7.5 gallons per minute (GPM). Two thermocouples, type T, (Thot-1, Thot-2) and two pressure sensors (Phot-1, Phot-2) are installed at inlet and outlet of the heat exchanger to measure the temperature and pressure. XV-1 and XV-3 are utilized as an air vent to relieve any air bubbles initially developed in the system. The four thermocouples, type $\mathrm{K}$, (TZ-1 to TZ-4) are placed along one of the tubes of the heat exchanger to extract more valuable information.

The shell side of the system is an open loop system with the water supply varied between 0 to 7.5 GPM from the facility internal plumbing. A hose transports the water to the shell side of the HX. This water flow rate (Fcold) is measured by an identical flow meter. The inlet and outlet temperatures are measured by thermocouples Tcold-1 and Tcold-2, respectively, on the shell side of the heat exchanger.

To increase the fouling rate naturally, Kaolin clay is added to the water in the tube side of the exchanger. Kaolin, produced by Thiele Kaolin Company, is appropriated for the small-scale shell and tube heat exchanger. The particle size is less than 2 micro millimeters with 98 percent of dry clay 
tested at 20 percent solids and $\mathrm{pH}$ 6.8. The concentration of Kaolin in the heat exchanger is approximately 2,800 ppm.

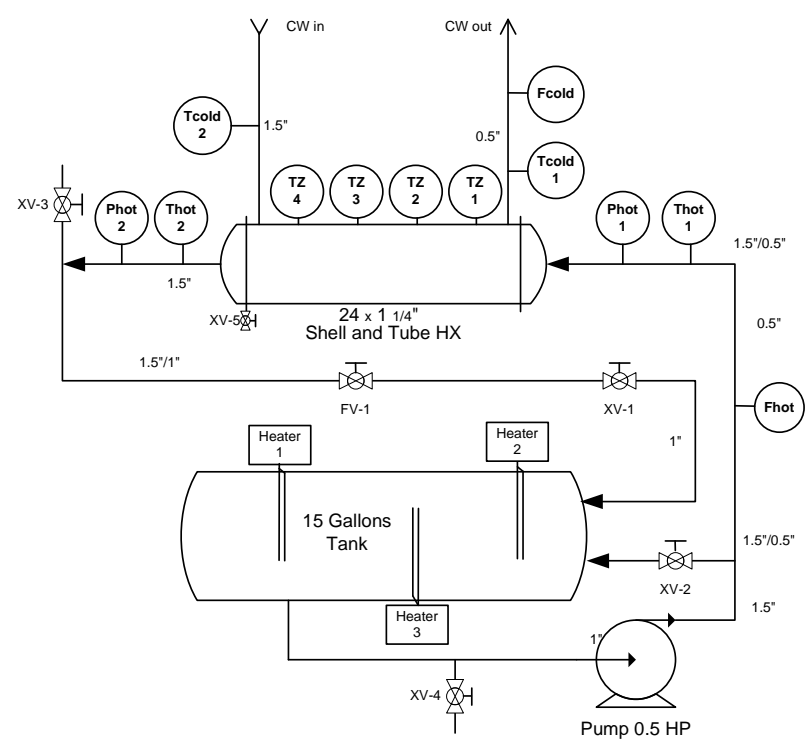

Figure 4. P\&ID of Heat Exchanger Test Bed

\section{RESULTS AND ANALYSIS}

The first part of this section presents the fouling prediction using AAKR models. The fouling data are then used to establish the HX degradation parameters in the GPM to predict the RUL

\subsection{Fouling prediction}

The heat exchanger was operated under accelerated fouling (due to Kaolin) until a steady-state is reached. The first experiment was run for 250 hours. After cleaning, the second experiment lasted 380 hours. In the second experiment, the process was disturbed by turning one heater off at the end of the data. The purpose is to test the ability of the model to detect any changes in the dynamic process. The fouling prediction is expected not to have this disturbance under normal operation. Figure 5 shows the plots of some of the process variables during accelerated fouling.

Several models based on different variables were investigated. The best prediction was from the group of variables based on the physical model, the overall thermal resistance (Equation 8), which consists of 5 variables, dtcold, dthot, LMTD, Fhot and Fcold. Fcold was removed because of constant value which is useless for the model. Figure 6 illustrates fouling data for this variable group.

In Figure 6, only one variable that is affected by the fouling is the LMTD which is used for fouling prediction. The process disturbance also has an influence on the LMTD.

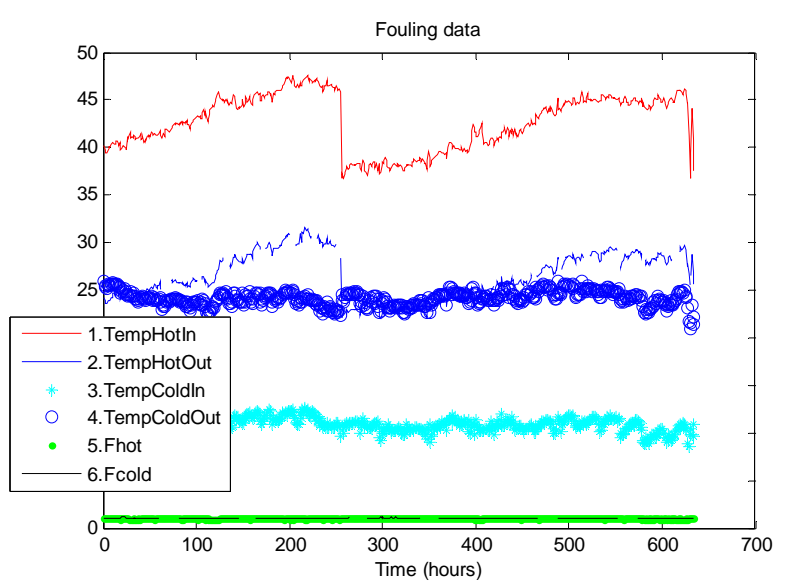

Figure 5. Fouling data

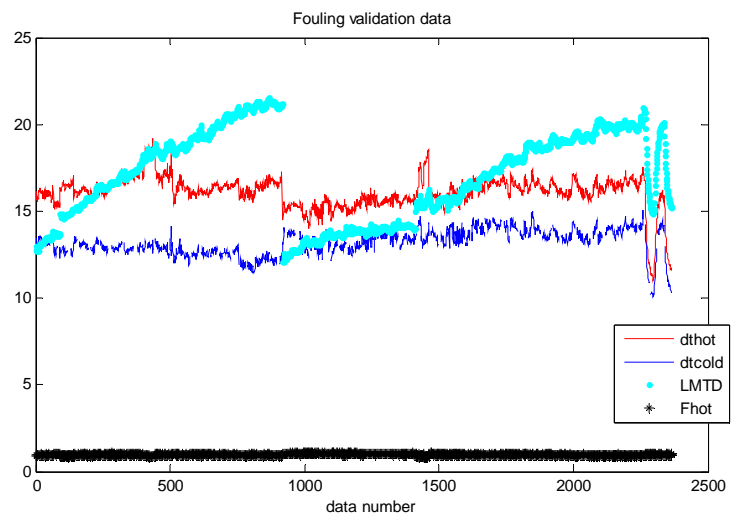

Figure 6. Fouling data for AAKR model development.

The results shown in Figure 7 indicate that the model can predict the fouling and has ability to detect the process disturbance at the end of fouling validation data. However, the prediction is not perfect since the fouling prediction is still under the influence of the process disturbance. The same experiment was run several times under the different operating condition and the prediction data will be then used in the next section.
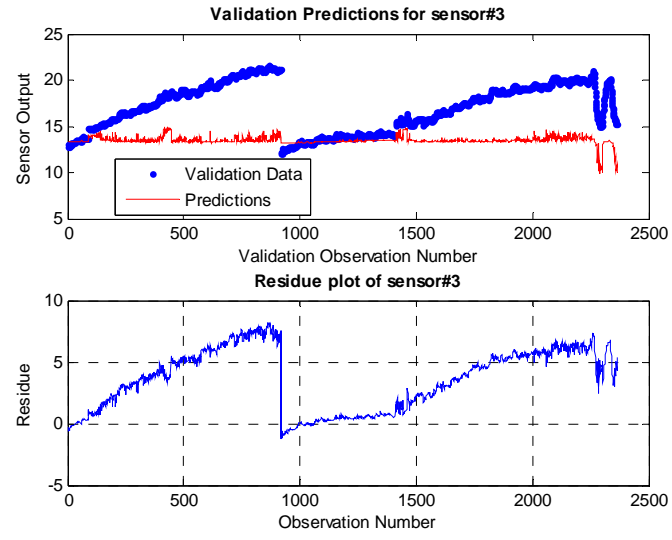

Figure 7. Prediction and Residual plot 


\subsection{Remaining Useful Life Prediction by GPM}

The fouling prediction data from the previous section are used as the degradation path in GPM. The degradation path is defined as the measure of fouling from the beginning to the steady state until the asymptotic behavior. In this study, degradation paths which were from the AAKR model prediction and also simulation were scaled from zero to one hundred percent. Figure 8 presents the degradation paths used in the GPM model for two different conditions.

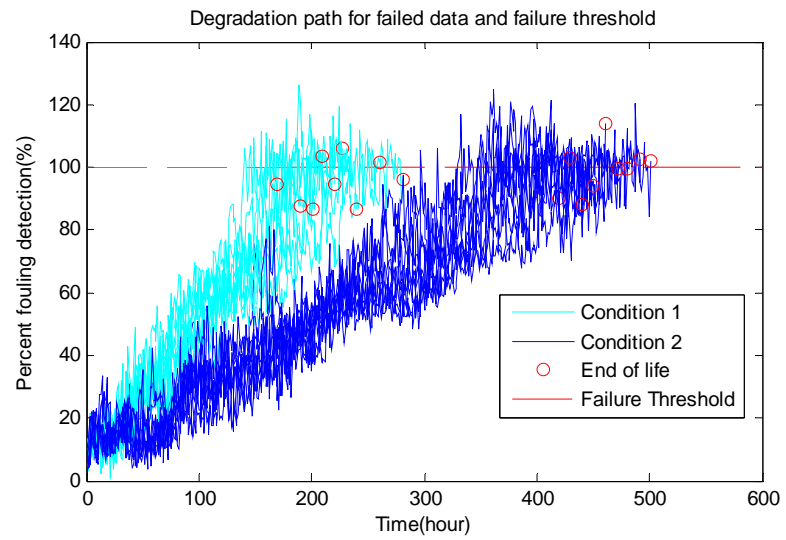

Figure 8. Degradation path from fouling prediction

Figure 8 shows that there are two groups of degradation paths. The first group consisting of 9 paths was operated with 1 GPM flow rate at both primary and secondary side. Nine degradation paths in the second group were operated with 3 GPM flow rate at primary side and 1 GPM at secondary side. The failure threshold is defined as the steady state of the fouling deposition or at $100 \%$. In fact, the experiments were not stopped immediately after fouling reached $100 \%$, but were continued for a period of time to ensure that the fouling reached the steady state.

The degradation measures were fit with the parametric models through the linear regression process. In this research, various models such as linear, exponential, quadratic were investigated. The model accuracy is defined by the average of Mean Square Error (MSE) of the model prediction for those degradation paths. Table 1 presents the result of the model fitting from both fouling condition.

Table 1. Model Fitting and Mean Square Error

\begin{tabular}{|c|c|c|}
\hline \multirow{2}{*}{ Model } & \multicolumn{2}{|c|}{ MSE } \\
\cline { 2 - 3 } & Condition 1 & Condition 2 \\
\hline Linear & 48.9 & 67.5 \\
\hline Quadratic & 49.0 & $\mathbf{6 7 . 1}$ \\
\hline Cubic & $\mathbf{4 7 . 5}$ & 72.0 \\
\hline Exponential & 168.3 & 179.1 \\
\hline
\end{tabular}

Next, the GPM with Bayesian updating was applied for unfailed fouling data to determine the RUL. The critical failure threshold and essential parameters such as regression coefficient and noise estimation from the failed data were used to determine the time to failure (TTF). The TTF is the time that the degradation measure reaches the critical threshold and the RUL is the period between the current time to TTF.

It is assumed that unfailed fouling data act like the prior degradation model. Those data will be used to generate the path and extrapolate to the failure threshold. Since the actual failure times for these units are known, the accuracy of the model can be obtained. See Figure 9 for the example of unfailed fouling degradation paths from both conditions.

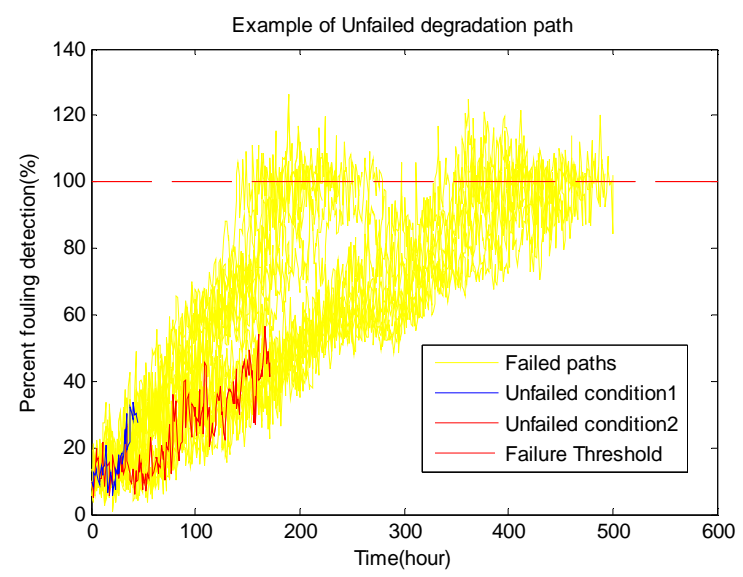

Figure 9. Example of unfailed fouling degradation paths

The RUL prediction was calculated using different parametric models such as linear, quadratic for each operating condition. The result was compared with the RUL prediction without reference to the operating condition. See Table 2 for a summary of results.

Table 2. TTF Prediction and Error

\begin{tabular}{|c|c|c|c|c|c|c|}
\hline \multirow{2}{*}{$\begin{array}{l}\text { Unfail } \\
\text { Path } \\
\text { number }\end{array}$} & \multirow{2}{*}{$\begin{array}{l}\text { Current } \\
\text { time }\end{array}$} & \multirow{2}{*}{$\begin{array}{c}\text { Actual } \\
\text { TTF }\end{array}$} & \multicolumn{4}{|c|}{ Predicted TTF [hours] } \\
\hline & & & $\begin{array}{c}\text { With } \\
\text { Stress } \\
\text { concern }\end{array}$ & Error & $\begin{array}{c}\mathrm{W} / \mathrm{O} \\
\text { Stress } \\
\text { concern }\end{array}$ & Error \\
\hline 1 & 45 & 150 & 129.8 & 13.5 & 200 & 33 \\
\hline 2 & 70 & 188 & 153.8 & 18.6 & 229 & 21 \\
\hline 3 & 83 & 206 & 193.7 & 5.9 & 262 & 27 \\
\hline 4 & 135 & 400 & 464.3 & 16.1 & 500 & 25 \\
\hline 5 & 172 & 414 & 404.5 & 2.3 & 425 & 3 \\
\hline 6 & 210 & 459 & 445.2 & 3.1 & 473 & 3 \\
\hline \multicolumn{3}{|c|}{ Average Error } & & 9.8 & & 18.6 \\
\hline
\end{tabular}

The results in Table 2 show that the GPM with Bayesian updating works well for this application. The average error 
is approximately 9.8 hours. However, it shows that knowledge of the stress or operating condition is important for improved prediction accuracy. The average error from the GPM with a separate operating condition half that of the GPM without knowledge of the operating condition. This example shows that understanding future operational stresses is extremely important for the accurate RUL predictive capabilities. Additional, understanding the relationship between the stresses and RUL estimates could be used to guide operations in order to survive to a maintenance opportunity.

\section{CONCLUDING REMARKS}

This research consists of two major parts: 1). monitoring the HX fouling using an AAKR model, and 2). predicting the RUL using a GPM with Bayesian updating. The data used in the analysis were collected from an experimental HX test bed which was operated with various operating conditions leading to different fouling rates.

The AAKR model developments were used for fouling prediction. The process disturbance was put into the validation data in order to test its effect on fouling prediction performance. Several models based on different measured and calculated variables were tested. The best model, which had the least influence from the process change, was from the model developed by the group of variables based on the physical model using overall thermal resistance.

A GPM with Bayesian updating, was developed and applied for the RUL prediction of fouling in the HX using the degradation data from the AAKR model. The result shows that this method is appropriate for use in the HX RUL application, which provides usable accuracy and errors of less than $10 \%$. Furthermore, this research shows the importance of understanding future stress conditions to achieve high accuracy prognostic predictions.

\section{ACKNOWLEDGMENTS}

This research is being performed using funding received from the DOE Office of Nuclear Energy's Nuclear Energy University Programs under grant DE-AC07-05IDPS0707ID14517 with the University of Tennessee.

\section{NOMENCLATURE}

AAKR Autoassociative Kernel Regression

GPM General Path Model

HX heat exchanger

LMTD Log Mean Temperature Difference

$R_{f} \quad$ the rate of fouling, fouling resistance or fouling factor

RUL Remaining Useful Life

TTF Time to Failure
U Overall heat transfer coefficient

\section{REFERENCES}

Lu C. J., \& W. Q. Meeker, Using degradation measures to estimate a Time-to-Failure distribution, Department of Statistics and Center for Nondestructive Evaluation, Iowa State University, May 1983.

Epstein, N.,"General Thermal Fouling Models," L.F. Melo, T.R. Bott and C.A. Bernardo, eds., Kluwer Academic Publishers, Dordrecht, The Netherlands, pp. 15-30, 1988.

Holman, J. P., Heat Transfer, 5th Edition, McGraw-Hill, New York, 1981.

Ingimundardóttir H., \& S. Lalot, "Detection of fouling in a cross-flow heat exchanger using wavelets" International Conference on Heat exchanger Fouling and Cleaning, 2009.

Hines J. W., \& J. Coble, "Applying the General Path Model to Estimation of Remaining Useful Life," International Journal of Prognostics and Health Monitoring (IJPHM), 2010 .

Hines, J. W., \& J. Coble, Process and Equipment Prognostics Toolbox, Tutorial version 1.0, University of Tennessee, 2011.

Hines J. W. \& D. Garvey, Process and Equipment Monitoring Toolbox Tutorial, Nuclear Engineering Department, University of Tennessee, 2006.

Hines J. W. \& A. Usynin, "MSET Performance Optimization Through Regularization", Nuclear Engineering and Technology, Vol. 37, No. 2, April 2005, pp 177-184.

Hines J. W., D. Garvey, \& R. Seibert, "On-Line Sensor Calibration Monitoring Challenges and Effective Monte Carlo Based Uncertainty Estimation," The University of Tennessee, 2003.

Steinhagen, R, H.M. Steinhagen, \& K. Maani, "Problems and Costs due to Heat Exchanger Fouling in New Zealand Industry," Heat Transfer Engineering, Vol. 14, No. 1, pp. 19-30, 1993.

Lingfang S., et al., "Research on the fouling prediction of heat exchanger based on support vector machine", International Conference on Intelligent Computation Technology and Automation (ICICTA), 2008.

Katsikis, V. N., "MATLAB- a fundamental tool for scientific computing and engineering application," Vol. 3, Chapter 3, Intech, September 2012.

Upadhyaya, B. R., J. W. Hines, et al., On-Line Monitoring and Diagnostics of the Integrity of Nuclear Plant Steam Generators and Heat Exchangers, Final Report: Volume 1, Experimental and Hybrid Modeling Approach for Monitoring Heat Exchanger System Performance, prepared for the DOE-NEER Program by the University of Tennessee, Knoxville, Report No. DE-FG0701ID14114/UTNE-07, September 2004. 
Nesta, J., "Reduce fouling in shell-and-tube heat exchangers," Hydrocarbon Processing, pp. 77-82, July 2004.

\section{BIOGRAPHIES}

Tutpol Ardsomang is pursuing his MS degree in Reliability and Maintainability Engineering with Industrial and Information engineering concentration from the University of Tennessee, Knoxville. He received his Bachelors in Instrumentation Engineering from the King Mongkut's Institute of Technology Ladkrabang and worked for 6 years with SCG chemical Co., Ltd in Thailand. He is now conducting research about the heat exchanger fouling prediction using the empirical modeling and prognostic techniques under Dr. Hines's.

Dr. J. Wesley Hines currently the Nuclear Engineering Department Head at the University of Tennessee. He is a Professor of Nuclear Engineering and the director of the Reliability and Maintainability Engineering program. He is a past nuclear qualified submarine officer and received an MBA and both an MS and Ph.D. in Nuclear Engineering from The Ohio State University. He has been with the University for 17 years. Dr. Hines teaches and conducts research in artificial intelligence and advanced statistical techniques applied to process diagnostics, condition based maintenance, and prognostics. He has authored over 250 papers and has several patents in the area of advanced process monitoring and prognostics techniques.

Dr. Belle R. Upadhyaya received the Ph.D. degree in Engineering Sciences (Systems Science) from the University of California at San Diego. He is currently a professor of Nuclear Engineering at The University of Tennessee, Knoxville. His research and teaching interests include instrumentation and controls, reactor dynamics, advanced digital signal processing, power and process plant monitoring and diagnosis, autonomous and fault-tolerant control of small modular reactors, nuclear desalination, sensor placement strategies, accelerated aging of detectors and equipment, and reliability and maintainability engineering. He has published over 320 articles in scientific journals and conference proceedings, chapters in handbooks, and is the author or co-author of over 130 research reports. Dr. Upadhyaya is an elected Fellow of the American Nuclear Society (ANS) and an elected Fellow of the International Society of Automation (ISA) 